\title{
Changes of deceleration and acceleration capacity of heart rate in patients with acute hemispheric ischemic stroke
}

\author{
Yan-Hong $\mathrm{Xu}^{\prime}$ \\ Xing-De Wang ${ }^{2}$ \\ Jia-Jun Yang' \\ Li Zhou ${ }^{2}$ \\ Yong-Chao Pan' \\ 'Department of Neurology, \\ ${ }^{2}$ Department of Cardiology, Shangha \\ Jiao Tong University Affiliated Sixth \\ People's Hospital, Shanghai, People's \\ Republic of China
}

This article was published in the following Dove Press journal:

Clinical Interventions in Aging

II March 2016

Number of times this article has been viewed

Correspondence: Jia-Jun Yang

Department of Neurology, Shanghai Jiao

Tong University Affiliated Sixth People's

Hospital, 600 Yishan Road, Shanghai

200233, People's Republic of China

Tel/fax +86 2l 38297763

Email yangjiajunfzy@sina.com

Xing-De Wang

Department of Cardiology, Shanghai Jiao Tong University Affiliated Sixth People's

Hospital, 600 Yishan Road, Shanghai

200233, People's Republic of China

Tel +86 2l 38297277

Emailwxdwsz8@163.com
Background and purpose: Autonomic dysfunction is common after stroke, which is correlated with unfavorable outcome. Phase-rectified signal averaging is a newly developed technique for assessing cardiac autonomic function, by detecting sympathetic and vagal nerve activity separately through calculating acceleration capacity (AC) and deceleration capacity (DC) of heart rate. In this study, we used this technique for the first time to investigate the cardiac autonomic function of patients with acute hemispheric ischemic stroke.

Methods: A 24-hour Holter monitoring was performed in 63 patients with first-ever acute ischemic stroke in hemisphere and sinus rhythm, as well as in 50 controls with high risk of stroke. DC, AC, heart rate variability parameters, standard deviation of all normal-to-normal intervals (SDNN), and square root of the mean of the sum of the squares of differences between adjacent normalto-normal intervals (RMSSD) were calculated. The National Institutes of Health Stroke Scale (NIHSS) was used to assess the severity of stroke. We analyzed the changes of DC, AC, SDNN, and RMSSD and also studied the correlations between these parameters and NIHSS scores.

Results: The R-R ( $\mathrm{R}$ wave to $\mathrm{R}$ wave on electrocardiogram) intervals, $\mathrm{DC}, \mathrm{AC}$, and SDNN in the cerebral infarction group were lower than those in controls $(P=0.003, P=0.002, P=0.006$, and $P=0.043$ ), but the difference of RMSSD and the $D$-value and ratio between absolute value of $\mathrm{AC}(|\mathrm{AC}|)$ and DC were not statistically significant compared with those in controls. The DC of the infarction group was significantly correlated with $|\mathrm{AC}|$, SDNN, and RMSSD $(r=0.857$, $r=0.619$, and $r=0.358 ; P=0.000, P=0.000$, and $P=0.004)$. Correlation analysis also showed that DC, $|\mathrm{AC}|$, and SDNN were negatively correlated with NIHSS scores $(r=-0.279, r=-0.266$, and $r=-0.319 ; P=0.027, P=0.035$, and $P=0.011$ ).

Conclusion: Both DC and AC of heart rate decreased in patients with hemispheric infarction, reflecting a decrease in both vagal and sympathetic modulation. Both DC and AC were correlated with the severity of stroke.

Keywords: acute ischemic stroke, autonomic dysfunction, deceleration capacity of heart rate, acceleration capacity of heart rate, heart rate variability

\section{Introduction}

Stroke has become the leading cause of death in the People's Republic of China ${ }^{1}$ and the second most common cause of death worldwide. ${ }^{2}$ Cardiac autonomic dysfunction is common after stroke, which is correlated with unfavorable outcome. ${ }^{3,4}$ As an electrophysiological method, heart rate variability (HRV) has been used to assess the autonomic function for decades. Studies have shown that HRV is related to the severity of stroke ${ }^{5}$ and has some mortality predictive values. ${ }^{6}$ However, it cannot accurately distinguish the sympathetic and vagal nerve activity. Phase-rectified signal averaging 
(PRSA) is a newly developed technique for detecting cardiac autonomic function, which can quantitatively detect the function of vagal and sympathetic nerves by calculating the deceleration capacity (DC) and acceleration capacity (AC) of the heart, respectively. Its advantages have been shown in the field of cardiology. ${ }^{7}$ However, this method has not yet been used in the study of autonomic dysfunction after stroke. In this study, the changes in DC and AC of heart rate and other related parameters were studied in patients with acute cerebral infarction for the purpose of investigating their values in assessing the autonomic function of patients with stroke.

\section{Materials and methods Subjects}

A total of 63 patients (38 men and 25 women; mean age, $71 \pm 12$ years) with first-ever acute cerebral infarction and sinus rhythm were recruited within 72 hours after stroke. A magnetic resonance imaging (MRI) or computed tomography (CT) scan confirmed the infarction located in the cerebral hemispheres, with 35 in the left hemisphere and 28 in the right. Patients with manifestations of other nervous system lesions and patients with any other diseases or medication known to affect the autonomic nervous system were excluded. Patients with tumor, infection, previous heart, or pulmonary disease were also excluded.

A total of 50 controls ( 24 men and 26 women; mean age, $68 \pm 11$ years) were recruited from subjects with high risk of stroke but without history of stroke. Patients who had two or more of the following major risk factors, or one major risk factor, and two or more secondary risk factors were defined as patients with high risk of stroke. Major risk factors include 1) hypertension, 2) hyperlipidemia, 3) diabetes mellitus, 4) age $>50$ years, and 5) metabolic syndrome; secondary risk factors include 1) atrial fibrillation or heart disease, 2) smoking, 3) family history of stroke or heart attack, 4) obesity, 5) regular alcohol consumption, 6) lack of physical exercise, 7) dietary excess oil, 8) sleep apnea, 9) male, 10) often gums bleeding, teeth loose, or loss, 11) ischemic ophthalmopathy, and 12) sudden deafness. MRI was used in the control group to exclude acute infarction. The other exclusion criteria were the same as for the stroke group.

We obtained oral informed consent from both patients and controls or their clients. This study was approved by the ethics committee of Shanghai Jiao Tong University Affiliated Sixth People's Hospital. Sex, age, and combined basic diseases and conditions were not statistically different between the two groups (Table 1).
Table I Comparison between infarction patients and controls

\begin{tabular}{|c|c|c|c|}
\hline $\begin{array}{l}\text { Characteristicl } \\
\text { parameter }\end{array}$ & $\begin{array}{l}\text { Infarction } \\
\text { patients }(n=63)\end{array}$ & $\begin{array}{l}\text { Controls } \\
(n=50)\end{array}$ & $P$-value \\
\hline Male, n (\%) & $38(60.3)$ & $24(48)$ & 0.191 \\
\hline Age, years & $70.70 \pm 11.92$ & $68.24 \pm 10.66$ & 0.258 \\
\hline Hypertension, n (\%) & $50(79.4)$ & $35(70.0)$ & 0.252 \\
\hline Diabetes mellitus, $\mathrm{n}(\%)$ & $21(33.3)$ & $12(24.0)$ & 0.278 \\
\hline Hyperlipidemia, n (\%) & $18(28.6)$ & $16(32.0)$ & 0.693 \\
\hline Smoking, n (\%) & 27 (42.9) & $20(40.0)$ & 0.760 \\
\hline $\begin{array}{l}\text { Regular alcohol } \\
\text { consumption, n (\%) }\end{array}$ & $4(6.3)$ & $5(10.0)$ & 0.506 \\
\hline Body mass index, $\mathrm{kg} / \mathrm{m}^{2}$ & $25.2 \pm 4.5$ & $24.7 \pm 3.9$ & 0.537 \\
\hline NIHSS & $6.52 \pm 5.68$ & - & - \\
\hline$R-R$ intervals, $m s$ & $865.3 \pm 132.9$ & $931.1 \pm 90.8$ & 0.003 \\
\hline $\mathrm{DC}, \mathrm{ms}$ & $5.55 \pm 1.97$ & $6.66 \pm 1.63$ & 0.002 \\
\hline $\mathrm{AC}, \mathrm{ms}$ & $-6.05 \pm 2.02$ & $-7.06 \pm 1.77$ & 0.006 \\
\hline SDNN, ms & $93.14 \pm 31.07$ & $103.92 \pm 23.14$ & 0.043 \\
\hline RMSSD, ms & $26.05 \pm 12.08$ & $28.24 \pm|2.6|$ & 0.349 \\
\hline$|A C|-D C$ & $0.50 \pm 1.07$ & $0.40 \pm 0.65$ & 0.563 \\
\hline$|A C| / D C$ & $1.159 \pm 0.529$ & $1.062 \pm 0.111$ & 0.157 \\
\hline
\end{tabular}

Abbreviations: AC, acceleration capacity; DC, deceleration capacity; NIHSS, National Institutes of Health Stroke Scale; RMSSD, square root of the mean of the sum of the squares of differences between adjacent normal-to-normal intervals; SDNN, standard deviation of all normal-to-normal intervals; $R-R$ intervals, $R$ wave to $R$ wave intervals on electrocardiogram; $|A C|$, absolute value of $A C$.

\section{Clinical materials}

MRI was used to obtain the side and location information of the infarction. If MRI was contraindicated or unable to be obtained, repeat CT scans were used in order to identify the exact location of infarction. The National Institutes of Health Stroke Scale (NIHSS) was used to record the severity of neurologic deficit. An experienced neurologist was in charge of this work.

\section{Detection of autonomic parameters}

All patients underwent a 24-hour Holter monitoring within 1 week after admission. The recordings were analyzed by a DMS Holter system (DMS300-3; Diagnostic Monitoring Software, Stateline, NV, USA). The interferences were excluded, and the events of arrhythmia were tagged artificially when playing back the recordings. The signal processing technique of PRSA was used to process sequences of $\mathrm{R}-\mathrm{R}$ intervals obtained from the Holter recordings. ${ }^{8}$ This technique provides separate characterizations of deceleration- and acceleration-related modulations, quantified by DC and AC. It is believed that DC reflects vagal activity and AC reflects sympathetic activity of the heart. The computer calculated DC and AC automatically. Meantime, standard deviation of all normal-to-normal intervals (SDNN) and square root of the mean of the sum of the squares of differences between adjacent normal-to-normal intervals (RMSSD) were also calculated through the 24-hour Holter recordings, which 
were widely used parameters of HRV and were considered to reflect overall variability and parasympathetic modulation of the heart, respectively. As the values of $\mathrm{AC}$ were negative, we used the absolute value of $\mathrm{AC}(|\mathrm{AC}|)$ in the analyses. We also calculated the $D$-value between $|\mathrm{AC}|$ and $\mathrm{DC}(|\mathrm{AC}|-\mathrm{DC})$ and the ratio of $|\mathrm{AC}|$ and $\mathrm{DC}(|\mathrm{AC}| / \mathrm{DC})$ to reflect the balance of sympathetic and parasympathetic modulation. The electrophysiological analysts remained blinded to the clinical data during the study period.

\section{Statistical analysis}

Mean values ( \pm standard deviation $[\mathrm{SD}]$ ) were calculated for continuous variables. Distributions of continuous variables were determined by the Shapiro-Wilk test. Group differences were assessed by Student's $t$-test or Mann-Whitney $U$-test. Comparisons of categorical variables were made using chisquare test or Fisher's exact test. Correlations between different autonomic parameters were calculated using Pearson's correlation test, and correlations between autonomic parameters and NIHSS scores were calculated using Spearman rank correlation test. Data analysis was performed using SPSS Version 17.0 (SPSS Inc., Chicago, IL, USA). A value of $P<0.05$ was considered statistically significant.

\section{Results}

\section{Comparison of autonomic parameters between infarction patients and controls}

Compared with controls, the R-R intervals, DC, AC, and $\mathrm{SDNN}$ of the infarction group were lower. But the difference of RMSSD, the $D$-value between $|\mathrm{AC}|$ and $\mathrm{DC}(|\mathrm{AC}|-\mathrm{DC})$, and the ratio of $|\mathrm{AC}|$ and $\mathrm{DC}(|\mathrm{AC}| / \mathrm{DC})$ were not statistically significant compared with controls (Table 1).

\section{Comparison between left and right hemispheric infarction}

Some autonomic parameters of patients with the right hemispheric infarction were slightly lower than that of the left, but the differences were not statistically significant (Table 2).

\section{Correlation analysis}

In the infarction group, DC correlated significantly with $|\mathrm{AC}|, \mathrm{SDNN}$, and RMSSD. In 63 patients with stroke, NIHSS scores ranged from 0 to $21(6.52 \pm 5.68)$. There is a negative correlation between NIHSS and DC, $|\mathrm{AC}|$, and SDNN. No significant correlation was identified between NIHSS scores and RMSSD, the $D$-value between $|\mathrm{AC}|$ and $\mathrm{DC}$, and the ratio of $|\mathrm{AC}|$ and $\mathrm{DC}$ (Table 3; Figures 1-3).
Table 2 Comparison between left and right hemispheric infarction

\begin{tabular}{llll}
\hline Parameter & $\begin{array}{l}\text { Left infarction } \\
(\mathbf{n}=\mathbf{3 5})\end{array}$ & $\begin{array}{l}\text { Right infarction } \\
(\mathbf{n}=\mathbf{2 8})\end{array}$ & P-value \\
\hline NIHSS & $7.60 \pm 6.44$ & $5.18 \pm 4.30$ & 0.080 \\
R-R intervals, ms & $869.4 \pm 119.2$ & $860.2 \pm 150.5$ & 0.789 \\
DC, ms & $5.66 \pm 1.81$ & $5.42 \pm 2.19$ & 0.627 \\
AC, ms & $-6.30 \pm 2.00$ & $-5.75 \pm 2.05$ & 0.288 \\
SDNN, ms & $93.40 \pm 29.98$ & $92.82 \pm 32.94$ & 0.942 \\
RMSSD, ms & $27.49 \pm 12.91$ & $24.25 \pm 10.93$ & 0.295 \\
|AC|-DC & $0.633 \pm 0.962$ & $0.329 \pm 1.184$ & 0.265 \\
|AC|/DC & $1.132 \pm 0.243$ & $1.194 \pm 0.751$ & 0.646 \\
\hline
\end{tabular}

Abbreviations: AC, acceleration capacity; DC, deceleration capacity; NIHSS, National Institutes of Health Stroke Scale; RMSSD, square root of the mean of the sum of the squares of differences between adjacent normal-to-normal intervals; $S D N N$, standard deviation of all normal-to-normal intervals; $R-R$ intervals, $R$ wave to $R$ wave intervals on electrocardiogram; $|A C|$, absolute value of $A C$.

\section{Discussion}

Patients with stroke often complicate with cardiac autonomic dysfunction, which can lead to a variety of cardiac arrhythmias, $T$ wave change, myocardial infarction, and even sudden death. ${ }^{9}$ Cardiac involvement is an important cause of death after stroke. ${ }^{10}$ Sympathetic hyperactivity and decrease in parasympathetic activity caused by stroke may be the reason of arrhythmia and sudden death. Studies found that cardiac autonomic dysfunction was related to the location and severity of stroke $\mathrm{e}^{4,5}$ and may be related to the unfavorable outcome of stroke., ${ }^{6,11,12}$

HRV has been used to detect the cardiac autonomic modulation for decades. However, it cannot distinguish the sympathetic and vagus nerve activity. In addition, there is no HRV parameter directly reflecting sympathetic modulation. ${ }^{13}$ Plasma catecholamine concentrations are usually used to measure the function of sympathetic nerve. However, plasma catecholamine concentrations are affected by many factors, such as the release, distribution, metabolism, and excretion of amines ${ }^{14}$ and they are also affected by circadian rhythm. ${ }^{15}$

Table 3 Correlation analysis between parameters of infarction group

\begin{tabular}{llllll}
\hline Parameter & \multicolumn{2}{l}{ DC } & & \multicolumn{2}{l}{ NIHSS } \\
\cline { 2 - 3 } \cline { 5 - 6 } & $\boldsymbol{r}$ & $\boldsymbol{P}$-value & & $\boldsymbol{r}$ & $\boldsymbol{P}$-value \\
\hline DC, ms & - & - & -0.279 & 0.027 \\
|AC|, ms & 0.857 & 0.000 & & -0.266 & 0.035 \\
SDNN, ms & 0.619 & 0.000 & -0.319 & 0.011 \\
RMSSD, ms & 0.358 & 0.004 & -0.154 & 0.277 \\
|AC|-DC & - & - & -0.084 & 0.514 \\
|AC|/DC & - & - & -0.125 & 0.328 \\
\hline
\end{tabular}

Abbreviations: AC, acceleration capacity; DC, deceleration capacity; NIHSS, National Institutes of Health Stroke Scale; RMSSD, square root of the mean of the sum of the squares of differences between adjacent normal-to-normal intervals; SDNN, standard deviation of all normal-to-normal intervals; $|A C|$, absolute value of $A C$. 


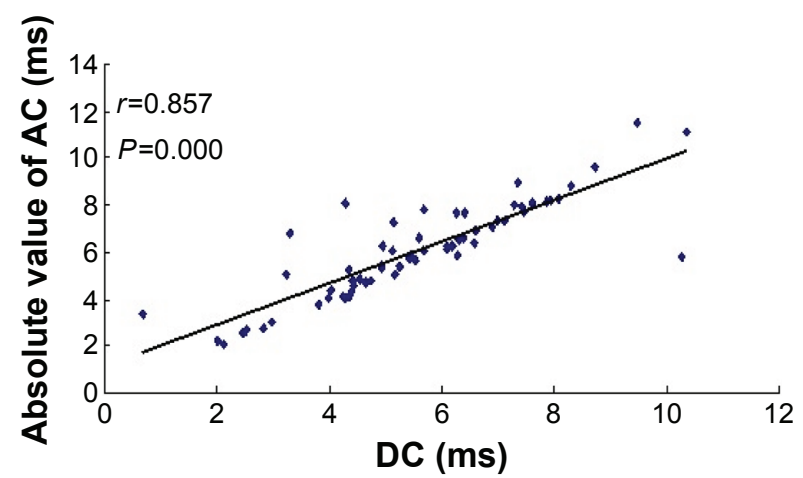

Figure I Correlation between DC and absolute value of AC.

Abbreviations: AC, acceleration capacity; DC, deceleration capacity.

PRSA is a new technique for detecting autonomic function. It can detect the AC and DC of the heart directly through analyzing the overall trend of 24-hour heart rate, and thus it can quantitatively detect the sympathetic activity and vagus activity, respectively, at the same time. ${ }^{7,8}$ This technique has been used mainly in the field of cardiovascular medicine, but not yet in the analysis of autonomic dysfunction after stroke.

In our study, we found that DC and AC as well as SDNN were all lower than controls, reflecting both sympathetic and vagal modulation loss in patients with hemispheric infarction. $\mathrm{DC}$ and $\mathrm{AC}$ were correlated with each other strongly, and the $D$-value and the ratio of absolute value of AC and DC had no significant difference compared with controls, reflecting that there was no major change in sympathovagal balance in patients with stroke. The loss of parasympathetic modulation is consistent with HRV studies. ${ }^{3,16}$ However, the decrease in $\mathrm{AC}$ reflects a decrease in sympathetic activity, which is inconsistent with studies concluding that the sympathetic activity of stroke increased through the detection of catecholamine concentrations. ${ }^{16,17}$ In the clinic, increased blood pressure, heart rate, and other characteristics of enhanced sympathetic activity are common in patients with cerebral

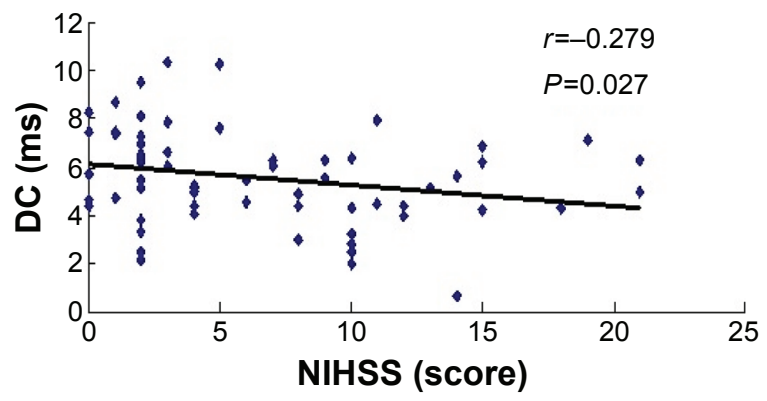

Figure 2 Correlation between NIHSS scores and DC.

Abbreviations: DC, deceleration capacity; NIHSS, National Institutes of Health Stroke Scale.

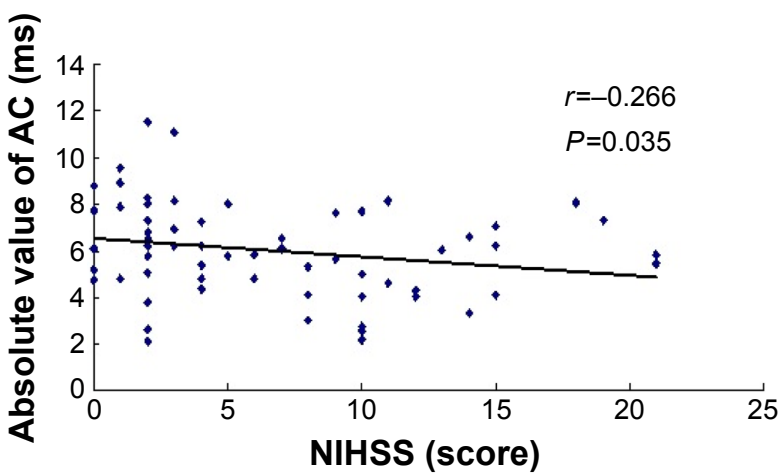

Figure 3 Correlation between NIHSS scores and absolute value of AC.

Abbreviations: AC, acceleration capacity; NIHSS, National Institutes of Health Stroke Scale.

infarction. In our study, we also found that R-R intervals of patients with infarction were shorter than those in controls, which might be a reflection of sympathetic hyperactivity. Our findings demonstrate a phenomenon that both the vagal and sympathetic modulation decrease after stroke, but the combined effects of the two result in predominant sympathetic activity. This phenomenon could be explained by the fact that the effect of heart rate increasing by decreased vagal activity was larger than the effect of heart rate decreasing by decreased sympathetic activity. The sympathetic activity is relatively predominant rather than absolutely predominant.

The study results were not conclusive of whether the lateralization and location of infarction were associated with autonomic derangement. Most studies found that if the infarctions are located in the right hemisphere especially with insula involvement, the cardiac autonomic dysfunction was more pronounced. ${ }^{4,18,19}$ In this study, autonomic parameters of patients with left and right cerebral infarction were compared. Although the autonomic parameters of patients with right hemispheric infarction were slightly lower than those of the left hemisphere, the differences were not statistically significant. Due to limited sample size, patients with the insula involvement were not analyzed separately. A further study of a larger number of cases is needed to search for the effect of lateralization and location of infarction on the autonomic nervous system.

In this study, the NIHSS score was used to assess the severity of stroke. Correlation analysis showed that DC, absolute value of AC, and SDNN were negatively correlated with NIHSS scores, which indicates a higher risk of autonomic complications in patients with more severe stroke. ${ }^{5}$ Although significant correlations between autonomic parameters and NIHSS scores were found, the $r$ values were rather low. We assume that autonomic function is affected by many factors 
such as age, sex, and combined diseases, which do not influence the NIHSS scores directly. The infarction location and lateralization also have different effects on autonomic modulation and NIHSS scores.

Declining autonomic modulation predicts poor outcomes in many diseases, such as myocardial infarction, ${ }^{20}$ chronic heart failure, ${ }^{21}$ and ischemic stroke. ${ }^{6}$ Studies also showed that vagus nerve had protective effects. ${ }^{22,23}$ The decline of vagal modulation may decrease this protective effect. DC, which is a direct reflection of vagal modulation, has shown its value in predicting mortality after myocardial infarction. ${ }^{7}$ Our study has also shown a decline in DC in patients with hemispheric infarction. Further studies for the value of DC in prognosis prediction in patients with stroke are deserved.

\section{Conclusion}

Both DC and AC decrease in patients with acute hemispheric infarction, reflecting the loss of both parasympathetic and sympathetic modulation after stroke. The clinical manifestations of hyperactivity of sympathetic nerve after stroke are possibly a reflection of the relative increase of sympathetic activity caused by more decline of vagal activity. Both DC and $\mathrm{AC}$ were correlated negatively with the severity of stroke. For the protective effect of vagal nerve, a decline of vagal modulation might have some predictive values for unfavorable outcomes after stroke. Further studies for the predictive value of DC and AC for the prognosis of stroke are deserved.

\section{Acknowledgments}

The authors sincerely thank the participants of this study for their cooperation. This study was supported by grants from the Science and Technology Foundation of Shanghai (14401970303).

\section{Disclosure}

The authors report no conflicts of interest in this work.

\section{References}

1. Liu L, Wang D, Wong KS, Wang Y. Stroke and stroke care in China: huge burden, significant workload, and a national priority. Stroke. 2011;42(12):3651-3654.

2. Lozano R, Naghavi M, Foreman K, et al. Global and regional mortality from 235 causes of death for 20 age groups in 1990 and 2010: a systematic analysis for the Global Burden of Disease Study 2010. Lancet. 2012;380(9859):2095-2128.

3. Korpelainen JT, Sotaniemi KA, Huikuri HV, Myllyä VV. Abnormal heart rate variability as a manifestation of autonomic dysfunction in hemispheric brain infarction. Stroke. 1996;27(11):2059-2063.
4. Tokgozoglu SL, Batur MK, Top uoglu MA, Saribas O, Kes S, Oto A. Effects of stroke localization on cardiac autonomic balance and sudden death. Stroke. 1999;30(7):1307-1311.

5. Hilz MJ, Moeller S, Akhundova A, et al. High NIHSS values predict impairment of cardiovascular autonomic control. Stroke. 2011;42(6): $1528-1533$

6. Mäkikallio AM, Mäkikallio TH, Korpelainen JT, Sotaniemi KA, Huikuri HV, Myllyä VV. Heart rate dynamics predict poststroke mortality. Neurology. 2004;62(10):1822-1826.

7. Bauer A, Schmidt G, Kantelhardt JW, et al. Deceleration capacity of heart rate as a predictor of mortality after myocardial infarction: cohort study. Lancet. 2006;367(9523):1674-1681.

8. Bauer A, Kantelhardt JW, Bunde A, Malik M, Schneider R, Schmidt G. Phase-rectified signal averaging detects quasiperiodicities in nonstationary data. Physica A. 2006;364:423-434.

9. Ay H, Koroshetz WJ, Benner T, et al. Neuroanatomic correlates of stroke-related myocardial injury. Neurology. 2006;66(9):1325-1329.

10. Prosser J, MacGregor L, Lees KR, et al; VISTA Investigators. Predictors of early cardiac morbidity and mortality after ischemic stroke. Stroke. 2007;38(8):2295-2302.

11. Colivicchi F, Bassi A, Santini M, Caltagirone C. Prognostic implications of right-sided insular damage, cardiac autonomic derangement, and arrhythmias after acute ischemic stroke. Stroke. 2005;36(8): $1710-1715$.

12. Bassi A, Colivicchi F, Santini M, Caltagirone C. Cardiac autonomic dysfunction and functional outcome after ischaemic stroke. Eur $J$ Neurol. 2007;14(8):917-922.

13. Cygankiewicz I, Zareba W. Heart rate variability. Handb Clin Neurol. 2013;117:379-393.

14. Esler M, Jennings G, Lambert G, Meredith I, Horne M, Eisenhofer G. Overflow of catecholamine neurotransmitters to the circulation: source, fate, and functions. Physiol Rev. 1990;70(4):963-985.

15. Schöfl C, Becker C, Prank K, von zur Mühlen A, Brabant G. Twentyfour-hour rhythms of plasma catecholamines and their relation to cardiovascular parameters in healthy young men. Eur J Endocrinol. 1997;137(6):675-683.

16. Ak1l E, Tamam Y, Ak1l MA, et al. Identifying autonomic nervous system dysfunction in acute cerebrovascular attack by assessments of heart rate variability and catecholamine levels. J Neurosci Rural Pract. 2015;6(2):145-150.

17. Myers MG, Norris JW, Hachniski VC, Sole MJ. Plasma norepinephrine in stroke. Stroke. 1981;12(2):200-204.

18. Colivicchi F, Bassi A, Santini M, Caltagirone C. Cardiac autonomic derangement and arrhythmias in right-sided stroke with insular involvement. Stroke. 2004;35(9):2094-2098.

19. Strittmatter M, Meyer S, Fischer C, Georg T, Schmitz B. Locationdependent patterns in cardio-autonomic dysfunction in ischaemic stroke. Eur Neurol. 2003;50(1):30-38.

20. La Rovere MT, Bigger JT Jr, Marcus FI, Mortara A, Schwartz PJ. Baroreflex sensitivity and heart-rate variability in prediction of total cardiac mortality after myocardial infarction. ATRAMI (autonomic tone and reflexes after myocardial infarction) investigators. Lancet. 1998;351(9101):478-484.

21. Nolan J, Batin PD, Andrews R, et al. Prospective study of heart rate variability and mortality in chronic heart failure: results of the United Kingdom heart failure evaluation and assessment of risk trial (UKheart). Circulation. 1998;98(15):1510-1516.

22. Ay I, Napadow V, Ay H. Electrical stimulation of the vagus nerve dermatome in the external ear is protective in rat cerebral ischemia. Brain Stimul. 2015;8(1):7-12.

23. Ng GA. Vagal modulation of cardiac ventricular arrhythmia. Exp Physiol. 2014;99(2):295-299. 


\section{Publish your work in this journal}

Clinical Interventions in Aging is an international, peer-reviewed journal focusing on evidence-based reports on the value or lack thereof of treatments intended to prevent or delay the onset of maladaptive correlates of aging in human beings. This journal is indexed on PubMed Central, MedLine,

CAS, Scopus and the Elsevier Bibliographic databases. The manuscript management system is completely online and includes a very quick and fair peer-review system, which is all easy to use. Visit http://www.dovepress. com/testimonials.php to read real quotes from published authors.

Submit your manuscript here: http://www.dovepress.com/clinical-interventions-in-aging-journal 